\title{
Lacunary Statistical Delta 2 2-Quasi Cauchy Double Sequences
}

\author{
A. G. K. Ali ${ }^{1}$, A. M Brono ${ }^{2}$, B. Sule \\ ${ }^{1}$ Department of Mathematics and Computer Science, Borno State University Maiduguri, Borno State, Nigeria \\ ${ }^{2}$ Department of Mathematical Sciences, University of Maiduguri, Borno State, Nigeria \\ ${ }^{3}$ Department of Mathematical Sciences, Federal University Dutsinma, Katsina State, Nigeria
}

\begin{tabular}{ll}
\hline ARTICLE INFO & ABSTRACT \\
\hline Published Online: & In this paper, we have introduced the extension of recently introduced notion of summability of \\
19 June 2021 & lacunary statistical delta 2 quasi Cauchy sequences to double sequences and established some essential \\
Corresponding Author: & results using analogy.
\end{tabular}

A. G. K. Ali

KEYWORDS: Lacunary statistical delta 2, Cauchy sequences, double sequences, summability.

2010 Mathematics subject classification: Primary 40F05, 40J05, 40G05

\section{INTRODUCTION}

The concept of statistical convergence was formally introduced by Fast [8] and Schoenberg [18] independently. Although statistical convergence was introduced over fifty years ago, it has become an active area of research in recent years. It has been applied in various areas such as summability theory (Fridy [9] and Salat [15]), topological groups (Cakalli [1], [4]), topological spaces (Maio and Kocinac [10]), locally convex spaces (Maddox [11]), measure theory (Cheng et al [5]), (Connor and Swardson [6]) and (Miller [12]), Fuzzy Mathematics (Nuray and Savas [14] and Savas [25]). In recent years generalization of statistical convergence has appeared in the study of strong summability and the structure of ideals of bounded functions, (Connor and Swardson [7]). Mursaleen and Edely [13] extended the notion of statistical convergence of single sequence to double sequences. Fridy and Orhan [9] introduced the concept of lacunary statistical convergence of single sequence. Savas and Patterson [17] extended the concept of lacunary statistical convergence of single sequence to double sequences. Yildiz [19] introduced the concept of lacunary statistical delta 2 quasi Cauchy sequences. In this paper we have further extended this concept to double sequences and established some essential results using analogy.

Definition 1.1: ([13]): A real double sequence $x=\left(x_{j k}\right)$ is statistically convergent to a number $l$ if for each $\varepsilon>0$, the set

$$
\left\{(j, k), j \leq n \text { and } k \leq m:\left|x_{j k}-l\right| \geq \varepsilon\right\}
$$

has double natural density zero. In this case we write $s t_{2}-\lim _{j k} x_{j k}=l$ and we denote the set of all statistically convergent double sequences by $s t_{2}$.

Definition $1.2([17])$. The double sequence $\theta_{r, s}=\left(j_{r}, k_{s}\right)$ is called double lacunary if there exist two increasing sequences of integers such that $j_{0}=0, h_{r}=j_{r}-j_{r-1} \rightarrow \infty$ as $r \rightarrow \infty$ and $k_{0}=0, h_{s}=k_{s}-k_{s-1} \rightarrow \infty$ as $s \rightarrow \infty$. Let $j_{r, s}=j_{r} k_{s}, h_{r, s}=h_{r} \overline{h_{s}}$ and $\theta_{r, s}$ is determined by $I_{r, s}=\left\{(j, k): j_{r-1}<j \leq j_{r}\right.$ and $\left.k_{s-1}<k \leq k_{s}\right\}, q_{r}=\frac{j_{r}}{j_{r-1}}, \overline{q_{s}}=\frac{k_{s}}{k_{s-1}}$ and $q_{r, s}=q_{r} \overline{q_{s}}$.

Definition 1.3 ([17]): Let $\theta_{r, s}$ be a double lacunary sequence, the double number sequence $x$ is double lacunary statistical convergent to $L$ provided that for every $\varepsilon>0$,

$$
\lim _{r, s} \frac{1}{h_{r, s}}\left|\left\{(j, k) \in I_{r, s}:\left|x_{j, k}-L\right| \geq \varepsilon\right\}\right|=0
$$

Definition 1.4 ([19]): A sequence $\left(x_{k}\right)$ in $\mathbb{R}$ is called lacunary statistically $\delta^{2}$ quasi-Cauchy, or $S_{\theta}-\delta^{2}$ quasi- Cauchy if the sequence $\left(\Delta^{2} x_{k}\right)$ is lacunary statistically quasi-Cauchy, i.e. 


$$
\lim _{r} \frac{1}{h_{r}}\left|\left\{k \in I_{r}:\left|\Delta^{3} x_{k}\right| \geq \varepsilon\right\}\right|=0
$$

For every positive real number $\varepsilon$, where $\Delta^{3} x_{k}=x_{k+3}-3 x_{k+2}+3 x_{k+1}-x_{k}$ for each positive integer $k$.

Definition 1.5 ([19]): A real valued function $f$ defined on a subset $A$ of $\mathbb{R}$ is called lacunary statistically $\delta^{2}$ ward continuous, or $S_{\theta}-\delta^{2}$ ward continuous on $A$ if it preserves lacunary statistically $\delta^{2}$ quasi-Cauchy sequences in $A$.

The set of lacunary statistical $\delta^{2}$ ward continuous functions on $A$ will be denoted by $\Delta^{3} C S_{\theta}(A)$.

\section{MAIN RESULTS}

A double sequence $\left(x_{j k}\right)$ in $\mathbb{R}$ is called double lacunary statistically 2-quasi-Cauchy if $S_{\theta_{r, s}}-\lim \Delta x_{j, k}=0$, where $\Delta x_{j, k}=$ $x_{j+1, k+1}-x_{j, k}$ for each positive integers $j, k$. The set of double lacunary statistically 2-quasi-Cauchy sequence will be denoted by $\Delta S_{\theta_{r, s}}$. If we put $\left|\Delta^{3} x_{j, k}\right|$ instead of $\left|\Delta^{2} x_{j, k}\right|$ in the above definition given in [3] we have:

Definition 2.1: A double sequence $\left(x_{j, k}\right)$ in $\mathbb{R}$ is called double lacunary statistically $\delta^{2}$ 2-quasi-Cauchy, or $S_{\theta_{r, s}}-\delta^{2}$ 2-quasiCauchy if the sequence $\left(\Delta^{3} x_{j, k}\right)$ is double lacunary statistically 2 -quasi-Cauchy, i.e.

$$
\lim _{r, s \rightarrow \infty} \frac{1}{h_{r, s}}\left|\left\{(j, k) \in I_{r, s}:\left|\Delta^{3} x_{j, k}\right| \geq \varepsilon\right\}\right|=0
$$

For every positive real number $\varepsilon$, where $\Delta^{3} x_{j, k}=x_{j+3, k+3}-3 x_{j+2, k+2}+3 x_{j+1, k+1}-x_{j, k}$ for each positive integers $j, k$.

We note that any $S_{\theta_{r, s}}-2$ quasi Cauchy double sequence is also $S_{\theta_{r, s}}-\delta^{2}-2$ quasi Cauchy, so is an oscillating sequence, so is a Cauchy double sequence, so is a convergent sequence, but the converses are not always true. Thus the inclusions

$C \subset \Delta S_{\theta_{r, S}} \subset \Delta^{3} S_{\theta_{r, s}}$, hold strictly, where $\Delta^{3} S_{\theta_{r, S}}$ denotes the set of $S_{\theta_{r, s}}-\delta^{2}-2$ quasi-Cauchy double sequences and $C$ denotes the set of Cauchy double sequence of points in $\mathbb{R}$.

Definition 2.2: A real valued function $f$ defined on a subset $A$ of $\mathbb{R}$ is called double lacunary statistically $\delta^{2}$ ward continuous, or $S_{\theta_{r, s}}-\delta^{2}$ ward continuous on $A$ if it preserves double lacunary statistically $\delta^{2}$ 2quasi-Cauchy double sequences in $A$.

The set of double lacunary statistical $\delta^{2}$ ward continuous functions on $A$ will be denoted by $\Delta^{3} C S_{\theta_{r, s}}(A)$.

Proposition 2.1: If $\left(x_{j, k}\right)$ and $\left(y_{j, k}\right)$ are double lacunary statistically $\delta^{2}$ 2quasi-Cauchy double sequences, then $\left(x_{j, k}+y_{j, k}\right)$ is a double lacunary statistically $\delta^{2}$ 2quasi-Cauchy double sequences.

Proof: Let $\left(x_{j, k}\right)$ and $\left(y_{j, k}\right)$ are double lacunary statistically $\delta^{2}$ 2quasi-Cauchy double sequences. To prove that $\left(x_{j, k}+y_{j, k}\right)$ is a double lacunary statistically $\delta^{2}$ 2quasi-Cauchy double sequence, take any $\varepsilon>0$. Then we have

$\lim _{r, s \rightarrow \infty} \frac{1}{h_{r, s}}\left|\left\{(j, k) \in I_{r, s}:\left|\Delta^{3} x_{j, k}\right| \geq \frac{\varepsilon}{2}\right\}\right|=0$ and $\lim _{r, s \rightarrow \infty} \frac{1}{h_{r, s}}\left|\left\{(j, k) \in I_{r, s}:\left|\Delta^{3} y_{j, k}\right| \geq \frac{\varepsilon}{2}\right\}\right|=0$.

Hence

This completes the proof.

$$
\lim _{r, s \rightarrow \infty} \frac{1}{h_{r, s}}\left|\left\{(j, k) \in I_{r, s}: \Delta^{3}\left(x_{j, k}+y_{j, k}\right)\right\} \geq \varepsilon\right|=0 .
$$

Proposition 2.2 Let $f \in \Delta^{3} C S_{\theta_{r, s}}(A), g \in \Delta^{3} C S_{\theta_{r, s}}(A)$ then $f+g \in \Delta^{3} C S_{\theta_{r, s}}(A)$.

Proof: Let $f \in \Delta^{3} C S_{\theta_{r, s}}(A), g \in \Delta^{3} C S_{\theta_{r, s}}(A)$. To prove that the sum $f+g$ is double lacunary statistically $\delta^{2}$ ward continuous on $A$, take any $\left(x_{j k}\right) \in \Delta^{3} S_{\theta_{r, s}}$. Then $\left(f\left(x_{j k}\right)\right) \in \Delta^{3} S_{\theta_{r, s}}$ and $\left(g\left(x_{j k}\right)\right) \in \Delta^{3} S_{\theta_{r, s}}$. Let $\varepsilon>0$ be given. Since $\left(f\left(x_{j k}\right)\right) \in \Delta^{3} S_{\theta_{r, s}}$ and $\left(g\left(x_{j k}\right)\right) \in \Delta^{3} S_{\theta_{r, s}}$, we have

$\lim _{r, s \rightarrow \infty} \frac{1}{h_{r, s}}\left|\left\{(j, k) \in I_{r, s}:\left|\Delta^{3} f\left(x_{j k}\right)\right| \geq \frac{\varepsilon}{2}\right\}\right|=0$ and $\lim _{r, s \rightarrow \infty} \frac{1}{h_{r, s}}\left|\left\{(j, k) \in I_{r, s}:\left|\Delta^{3} g\left(x_{j k}\right)\right| \geq \frac{\varepsilon}{2}\right\}\right|=0$.

Hence

This completes the proof.

$$
\lim _{r, s \rightarrow \infty} \frac{1}{h_{r, s}}\left|\left\{(j, k) \in I_{r, s}:\left|\Delta^{3}\left(f\left(x_{j k}\right)+g\left(x_{j k}\right)\right)\right| \geq \frac{\varepsilon}{2}\right\}\right|=0
$$

On the other hand, the product of a constant real number and $f \in \Delta^{3} C S_{\theta_{r, s}}$ is an element of $\Delta^{3} C S_{\theta_{r, s}}$.

Thus $\Delta^{3} C S_{\theta_{r, s}}$ is a vector space.

In connection with double lacunary statistically $\delta^{2}-2$ quasi-Cauchy double sequences and convergent sequences the problem arises to investigate the following types of continuity of functions on $\mathbb{R}$ :

$$
\begin{gathered}
\left(\delta^{2} S_{\theta_{r, s}} \delta^{2}\right):\left(x_{m, n}\right) \in \Delta^{3} S_{\theta_{r, s}} \Rightarrow\left(f\left(x_{m, n}\right)\right) \in \Delta^{3} S_{\theta_{r, s}} \\
\left(\delta^{2} S_{\theta_{r, s}} c\right):\left(x_{m, n}\right) \in \Delta^{3} S_{\theta_{r, s}} \Rightarrow\left(f\left(x_{m, n}\right)\right) \in c
\end{gathered}
$$




$$
\begin{gathered}
\left(\Delta S_{\theta_{r, s}}\right):\left(x_{m, n}\right) \in \Delta S_{\theta_{r, s}} \Rightarrow\left(f\left(x_{m, n}\right)\right) \in \Delta S_{\theta_{r, s}} \\
(c):\left(x_{m, n}\right) \in c \Rightarrow\left(f\left(x_{m, n}\right)\right) \in c \\
\left(S_{\theta_{r, s}}\right):\left(x_{m, n}\right) \in S_{\theta_{r, s}} \Rightarrow\left(f\left(x_{m, n}\right)\right) \in S_{\theta_{r, s}} \\
\left(c S_{\theta_{r, s}} \delta^{2}\right):\left(x_{m, n}\right) \in c \Rightarrow\left(f\left(x_{m, n}\right)\right) \in \Delta^{3} S_{\theta_{r, s}}
\end{gathered}
$$

We see that $\left(\delta^{2} S_{\theta_{r, s}} \delta^{2}\right)$ is double lacunary statistically $\delta^{2}$-ward continuity of $f,\left(S_{\theta_{r, s}}\right)$ is $S_{\theta_{r, s}}$-sequential continuity of $f$, and $(c)$ is ordinary continuity of $f$.bIt is easy to see that $\left(\delta^{2} S_{\theta_{r, s}} c\right)$ implies $\left(\delta^{2} S_{\theta_{r, s}} \delta^{2}\right)$, and $\left(\delta^{2} S_{\theta_{r, s}} \delta^{2}\right)$ does not imply $\left(\delta^{2} S_{\theta_{r, s}} c\right)$, and $\left(\delta^{2} S_{\theta_{r, s}} \delta^{2}\right)$ implies $\left(c S_{\theta_{r, S}} \delta^{2}\right)$, and $\left(c S_{\theta_{r, S}} \delta^{2}\right)$ does not imply $\left(\delta^{2} S_{\theta_{r, S}} \delta^{2}\right),\left(\delta^{2} S_{\theta_{r, s}} c\right)$ implies $(c)$, and (c) does not imply $\left(\delta^{2} S_{\theta_{r, s}} c\right)$.

Now we give the implication $\left(\delta^{2} S_{\theta_{r, s}} \delta^{2}\right)$ implies $\left(\Delta S_{\theta_{r, s}}\right)$.

Theorem 2.1: If $f \in \Delta^{3} C S_{\theta_{r, s}}(A)$, then $f \in \Delta C S_{\theta_{r, s}}(A)$.

Proof: Suppose that $f \in \Delta^{3} C S_{\theta_{r, s}}(A)$. Let $\left(x_{m, n}\right) \in \Delta S_{\theta_{r, s}}(A)$. Then the double sequence

$\left(x_{1,1}, x_{1,1}, x_{1,1}, x_{2,2}, x_{2,2}, x_{2,2}, \ldots, x_{m-1, n-1}, x_{m-1, n-1}, x_{m-1, n-1}, x_{m, n}, x_{m, n}, x_{m, n}\right)$ is in $\Delta S_{\theta_{r, s}}(A)$, so is in $\Delta^{2} S_{\theta_{r, s}}(A)$. Since $f$ is in $\Delta^{2} \operatorname{CS}_{\theta_{r, s}}(A)$, then the double sequence

$$
\begin{gathered}
\left(y_{m, n}\right)=\left(f\left(x_{1,1}\right), f\left(x_{1,1}\right), f\left(x_{1,1}\right), f\left(x_{2,2}\right), f\left(x_{2,2)}, f\left(x_{2,2}\right), \ldots, f\left(x_{m-1, n-1}\right), f\left(x_{m-1, n-1}\right),\right.\right. \\
\left.f\left(x_{m-1, n-1}\right), f\left(x_{m, n}\right), f\left(x_{m, n}\right), f\left(x_{m, n}\right)\right)
\end{gathered}
$$

is in $\Delta^{2} S_{\theta_{r, s}}(A)$. Then $\left(f\left(x_{m, n}\right)\right) \in \Delta S_{\theta_{r, s}}(A)$.

Corollary 2.1: If $f \in \Delta^{3} C S_{\theta_{r, s}}(A)$, then $f$ is continuous.

Proof: The proof follows immediately from the preceding theorem, so is omitted.

We note that any double lacunary statistically $\delta^{2}$ ward continuous function is $G$-sequentially continuous for any regular subsequential sequential method (see [2])

Theorem 2.2: If a real valued function $f$ is uniformly continuous on a subset $A$ of $\mathbb{R}$, then $\left(f\left(x_{m, n}\right)\right)$ is double lacunary statistically $\delta^{2}$ 2quasi-Cauchy double sequence of points in $A$.

Proof: Let $f$ be uniformly continuous on $A$. Take any 2quasi-Cauchy double sequence $\left(x_{m, n}\right)$ of points in $A$. Let $\varepsilon$ be any positive real number. Since $f$ is uniformly continuous, there exists $\delta>0$ such that $|f(x)-f(y)|<\frac{\varepsilon}{3}$ whenever $|x-y|<\delta$.

As $\left(x_{j, k}\right)$ is a 2quasi-Cauchy double sequence, for this $\delta$ there exist $m_{0}, n_{0} \in \mathbb{N}$ such that $\left|x_{j+1, k+1}-x_{j, k}\right|<\delta$ for $j, k \geq m_{0}, n_{0}$. Therefore $\left|f\left(x_{j+1, k+1}\right)-f\left(x_{j, k}\right)\right|<\frac{\varepsilon}{3}$ for $m, n \geq m_{0}, n_{0}$, so the number of indices of $j, k$ for which $\left|f\left(x_{j+1, k+1}\right)-f\left(x_{j, k}\right)\right| \geq \frac{\varepsilon}{3}$ is less than $m_{0}, n_{0}$. Hence

$$
\begin{aligned}
\lim _{r, s \rightarrow \infty} \frac{1}{h_{r, s}} \mid\{(j, k) & \left.\in I_{r, s}:\left|\Delta^{3} f\left(x_{j, k}\right)\right| \geq \varepsilon\right\} \mid \\
& =\lim _{r, s \rightarrow \infty} \frac{1}{h_{r, s}}\left|\left\{(j, k) \in I_{r, s}:\left|f\left(x_{j+3, k+3}\right)-3 f\left(x_{j+2, k+2}\right)+3 f\left(x_{j+1, k+1}\right)-f\left(x_{j, k}\right)\right| \geq \varepsilon\right\}\right| \\
& \leq \lim _{r, s} \frac{1}{h_{r, s}}\left|\left\{(j, k) \in I_{r, s}:\left|f\left(x_{j+3, k+3}\right)-f\left(x_{j+2, k+2}\right)\right| \geq \frac{\varepsilon}{3}\right\}\right| \\
& +\lim _{r, s} \frac{1}{h_{r, s}}\left|\left\{(j, k) \in I_{r, s}:\left|2 f\left(x_{j+2, k+2}\right)-2 f\left(x_{j+1, k+1}\right)\right| \geq \frac{\varepsilon}{3}\right\}\right| \\
& +\lim _{r, s} \frac{1}{h_{r, s}}\left|\left\{(j, k) \in I_{r, s}:\left|f\left(x_{j+1, k+1}\right)-f\left(x_{j, k}\right)\right| \geq \frac{\varepsilon}{3}\right\}\right| \leq \lim _{r, s \rightarrow \infty} \frac{m_{0} n_{0}}{h_{r, s}}+\lim _{r, s \rightarrow \infty} \frac{2 m_{0} n_{0}}{h_{r, s}}+\lim _{r, s \rightarrow \infty} \frac{m_{0} n_{0}}{h_{r, s}} \\
& =0+0+0=0 .
\end{aligned}
$$

This completes the proof.

Theorem 2.3: The uniform limit of double sequence of double lacunary statistically $\delta^{2}$ ward continuous functions is double lacunary statistically $\delta^{2}$ ward continuous.

Proof: Let $\left(f_{m, n}\right)$ be a double sequence of double lacunary statistically $\delta^{2}$ ward continuous functions on a subset $A$ of $\mathbb{R}$ and $\left(f_{m, n}\right)$ is uniformly convergent to a function $f$. To prove that $f$ is double lacunary statistically $\delta^{2}$ ward continuous on $A$, take a double lacunary statistically $\delta^{2}$ 2quasi-Cauchy double sequence $\left(x_{j, k}\right)$ of points in $A$, and let $\varepsilon$ be any positive real number. By the uniform convergence of $\left(f_{m, n}\right)$, there exist positive integers $m_{1}, n_{1}$ such that

$\left|f(x)-f_{j, k}(x)\right|<\frac{\varepsilon}{3}$ for $m, n \geq m_{1}, n_{1}$ and every $x \in A$. As $f_{m_{1}, n_{1}}$ is double lacunary statistically $\delta^{2}$ ward continuous on $A$, it follows that 
Now

$$
\lim _{r, s \rightarrow \infty} \frac{1}{h_{r, s}}\left|\left\{(j, k) \in I_{r, s}:\left|f_{m_{1} n_{1}}\left(x_{j+3, k+3}\right)-3 f_{m_{1} n_{1}}\left(x_{j+2, k+2}\right)+3 f_{m_{1} n_{1}}\left(x_{j+1, k+1}\right)-f_{m_{1} n_{1}}\left(x_{j, k}\right)\right| \geq \frac{\varepsilon}{3}\right\}\right|=0 .
$$

$$
\lim _{r, s \rightarrow \infty} \frac{1}{h_{r, s}}\left|\left\{(j, k) \in I_{r, s}:\left|f\left(x_{j+3, k+3}\right)-3 f\left(x_{j+2, k+2}\right)+3 f\left(x_{j+1, k+1}\right)-f\left(x_{j, k}\right)\right| \geq \varepsilon\right\}\right|
$$

$$
\begin{gathered}
\lim _{r, s \rightarrow \infty} \frac{1}{h_{r, s}} \mid\left\{(j, k) \in I_{r, s}: \mid f\left(x_{j+3, k+3}\right)-3 f\left(x_{j+2, k+2}\right)+3 f\left(x_{j+1, k+1}\right)-f\left(x_{j, k}\right)\right. \\
\left.-\left[f_{m_{1} n_{1}}\left(x_{j+3, k+3}\right)-3 f_{m_{1} n_{1}}\left(x_{j+2, k+2}\right)+3 f_{m_{1} n_{1}}\left(x_{j+1, k+1}\right)-f_{m_{1} n_{1}}\left(x_{j, k}\right)\right] \mid \geq \frac{\varepsilon}{3}\right\} \mid \\
\leq \lim _{r, s \rightarrow \infty} \frac{1}{h_{r, s}}\left|\left\{(j, k) \in I_{r, s}:\left|f\left(x_{j+3, k+3}\right)-f_{m_{1}, n_{1}}\left(x_{j+3, k+3}\right)\right| \geq \frac{\varepsilon}{3}\right\}\right| \\
\quad+\lim _{r, s \rightarrow \infty} \frac{1}{h_{r, s}}\left|\left\{(j, k) \in I_{r, s}:\left|-3 f\left(x_{j+2, k+2}\right)+3 f_{m_{1}, n_{1}}\left(x_{j+2, k+2}\right)\right| \geq \frac{\varepsilon}{3}\right\}\right| \\
+\lim _{r, s \rightarrow \infty} \frac{1}{h_{r, s}}\left|\left\{(j, k) \in I_{r, s}:\left|3 f\left(x_{j+1, k+1}\right)-3 f_{m_{1}, n_{1}}\left(x_{j+1, k+1}\right)\right| \geq \frac{\varepsilon}{3}\right\}\right| \\
\quad+\lim _{r, s \rightarrow \infty} \frac{1}{h_{r, s}}\left|\left\{(j, k) \in I_{r, s}:\left|f\left(x_{j, k}\right)-f_{m_{1}, n_{1}}\left(x_{j, k}\right)\right| \geq \frac{\varepsilon}{3}\right\}\right| \\
+\lim _{r, s \rightarrow \infty} \frac{1}{h_{r, s}}\left|\left\{(j, k) \in I_{r, s}:\left|f_{m_{1}, n_{1}}\left(x_{j+3, k+3}\right)-3 f_{m_{1}, n_{1}}\left(x_{j+2, k+2}\right)+3 f_{m_{1}, n_{1}}\left(x_{j+1, k+1}\right)-f_{m_{1}, n_{1}}\left(x_{j, k}\right)\right| \geq \frac{\varepsilon}{3}\right\}\right| \\
=0+0+0+0+0=0 .
\end{gathered}
$$

So $f$ preserves double lacunary statistically $\delta^{2}$ 2quasi-Cauchy double sequences. This completes the proof of the theorem.

Theorem 2.4: The set of double lacunary statistically $\delta^{2}$ ward continuous functions on a subset $A$ of $\mathbb{R}$ isva closed subset of the set of continuous functions on $A$.

Proof: Let $f$ be an element in the closure of the set of double lacunary statistically $\delta^{2}$ ward continuous functions on $A$. Then there exists a double sequence $\left(f_{m, n}\right)$ of points in the set of double lacunary statistically $\delta^{2}$ ward continuous functions such that $\lim _{m, n \rightarrow \infty} f_{m, n}=f$. To shows that $f$ is double lacunary statistically $\delta^{2}$ ward continuous, consider a double lacunary statistically $\delta^{2}$ 2quasi-Cauchy double sequence $\left(x_{j, k}\right)$ of points in $A$. Since $\left(f_{j, k}\right)$ converges to $f$, there exist positive integers $N_{1}, N_{2}$ such that for all $x \in A$ and for all $m, n \geq N_{1}, N_{2}$,

$\left|f_{j, k}(x)-f(x)\right|<\frac{\varepsilon}{12}$. As $f_{N_{1}, N_{2}}$ is double lacunary statistically $\delta^{2}$ ward continuous on $A$, we have that

$$
\begin{gathered}
\lim _{r, s \rightarrow \infty} \frac{1}{h_{r, s}}\left|\left\{(j, k) \in I_{r, s}:\left|f_{N_{1}, N_{2}}\left(x_{j+3, k+3}\right)-3 f_{N_{1}, N_{2}}\left(x_{j+2, k+2}\right)+3 f_{N_{1}, N_{2}}\left(x_{j+1, k+1}\right)-f_{N_{1}, N_{2}}\left(x_{j, k}\right)\right| \geq \frac{\varepsilon}{3}\right\}\right| \\
=0 .
\end{gathered}
$$

Now

$$
\begin{aligned}
\lim _{r, s \rightarrow \infty} \frac{1}{h_{r, s}} \mid\{(j, k) & \left.\in I_{r, s}:\left|f\left(x_{j+3, k+3}\right)-3 f\left(x_{j+2, k+2}\right)+3 f\left(x_{j+1, k+1}\right)-f\left(x_{j, k}\right)\right| \geq \varepsilon\right\} \mid \\
& =\lim _{r, s \rightarrow \infty} \frac{1}{h_{r, s}} \mid\{(j, k) \\
& \in I_{r, s}: \mid f\left(x_{j+3, k+3}\right)-3 f\left(x_{j+2, k+2}\right)+3 f\left(x_{j+1, k+1}\right)-f\left(x_{j, k}\right) \\
& \left.-\left[f_{N_{1}, N_{2}}\left(x_{j+3, k+3}\right)-3 f_{N_{1}, N_{2}}\left(x_{j+2, k+2}\right)+3 f_{N_{1}, N_{2}}\left(x_{j+1, k+1}\right)-f_{N_{1}, N_{2}}\left(x_{j, k}\right)\right] \mid \geq \frac{\varepsilon}{3}\right\} \mid \\
& \leq \lim _{r, s \rightarrow \infty} \frac{1}{h_{r, s}}\left|\left\{(j, k) \in I_{r, s}:\left|f\left(x_{j+3, k+3}\right)-f_{N_{1}, N_{2}}\left(x_{j+3, k+3}\right)\right| \geq \frac{\varepsilon}{3}\right\}\right| \\
& +\lim _{r, s \rightarrow \infty} \frac{1}{h_{r, s}}\left|\left\{(j, k) \in I_{r, s}:\left|-3 f\left(x_{j+2, k+2}\right)+3 f_{N_{1}, N_{2}}\left(x_{j+2, k+2}\right)\right| \geq \frac{\varepsilon}{3}\right\}\right| \\
& +\lim _{r, s \rightarrow \infty} \frac{1}{h_{r, s}}\left|\left\{(j, k) \in I_{r, s}:\left|3 f\left(x_{j+1, k+1}\right)-3 f_{N_{1}, N_{2}}\left(x_{j+1, k+1}\right)\right| \geq \frac{\varepsilon}{3}\right\}\right| \\
& +\lim _{r, s \rightarrow \infty} \frac{1}{h_{r, s}}\left|\left\{(j, k) \in I_{r, s}:\left|f\left(x_{j, k}\right)-f_{N_{1}, N_{2}}\left(x_{j, k}\right)\right| \geq \frac{\varepsilon}{3}\right\}\right| \\
& +\lim _{r, s \rightarrow \infty} \frac{1}{h_{r, s}}\left|\left\{(j, k) \in I_{r, s}:\left|f_{N_{1}, N_{2}}\left(x_{j+3, k+3}\right)-3 f_{N_{1}, N_{2}}\left(x_{j+2, k+2}\right)+3 f_{N_{1}, N_{2}}\left(x_{j+1, k+1}\right)-f_{N_{1}, N_{2}}\left(x_{j, k}\right)\right| \geq \frac{\varepsilon}{3}\right\}\right| \\
& =0+0+0+0+0=0 .
\end{aligned}
$$

Thus $f$ preserves double lacunary statistically $\delta^{2}$ 2quasi-Cauchy double sequences. This completes the proof of the theorem. 
Corollary 2.2: The set of double lacunary statistically $\delta^{2}$ ward continuous functions on a subset $A$ of $\mathbb{R}$ is complete as a subset of the set of continuous functions on $A$.

Theorem 2.5: The set of functions on a subset $A$ of $\mathbb{R}$ which map 2quasi-Cauchy double sequences to double lacunary statistically $\delta^{2}$ 2quasi-Cauchy double sequences is closed as a subset of the set of continuous functions on $A$.

Proof: It is easy to see that any function which maps 2quasi-Cauchy double sequence to double lacunary statistically $\delta^{2}$ 2quasiCauchy double sequences is continuous. Let $f$ be an element in the closure of the set of functions on $A$ which map 2quasi-Cauchy double sequences to double lacunary statistically $\delta^{2}$ 2quasi-Cauchy double sequences. Then there exists a double sequence $\left(f_{m, n}\right)$ of points in the set of functions on a subset $A$ of $\mathbb{R}$ which map 2quasi-Cauchy double sequences such that $\lim _{m, n} f_{m, n}=f$. To show that $f$ maps 2quasi-Cauchy double sequences to double lacunary statistically $\delta^{2}$ 2quasi-Cauchy double sequences, consider a 2quasi-Cauchy double sequence $\left(x_{j, k}\right)$ of points in $A$. Since $\left(f_{j, k}\right)$ converges to $f$, there exist positive integers $N_{1}, N_{2}$ such that for all $m, n \geq N_{1}, N_{2},\left|f_{j, k}(x)-f(x)\right|<\frac{\varepsilon}{3}$. As $f_{N_{1}, N_{2}}$ maps 2quasi-Cauchy double sequences to double lacunary statistically $\delta^{2}$ 2quasiCauchy double sequences, we have that

$$
\lim _{r, s \rightarrow \infty} \frac{1}{h_{r, s}}\left|\left\{(j, k) \in I_{r, s}:\left|f_{N_{1}, N_{2}}\left(x_{j+3, k+3}\right)-3 f_{N_{1}, N_{2}}\left(x_{j+2, k+2}\right)+3 f_{N_{1}, N_{2}}\left(x_{j+1, k+1}\right)-f_{N_{1}, N_{2}}\left(x_{j, k}\right)\right| \geq \frac{\varepsilon}{3}\right\}\right|=0 .
$$

Now

$$
\begin{aligned}
\lim _{r, s \rightarrow \infty} \frac{1}{h_{r, s}} \mid\{(j, k) & \left.\in I_{r, s}:\left|f\left(x_{j+3, k+3}\right)-3 f\left(x_{j+2, k+2}\right)+3 f\left(x_{j+1, k+1}\right)-f\left(x_{j, k}\right)\right| \geq \varepsilon\right\} \mid \\
& =\lim _{r, s \rightarrow \infty} \frac{1}{h_{r, s}} \mid\{(j, k) \\
& \in I_{r, s}: \mid f\left(x_{j+3, k+3}\right)-3 f\left(x_{j+2, k+2}\right)+3 f\left(x_{j+1, k+1}\right)-f\left(x_{j, k}\right) \\
& \left.-\left[f_{N_{1}, N_{2}}\left(x_{j+3, k+3}\right)-3 f_{N_{1}, N_{2}}\left(x_{j+2, k+2}\right)+3 f_{N_{1}, N_{2}}\left(x_{j+1, k+1}\right)-f_{N_{1}, N_{2}}\left(x_{j, k}\right)\right] \mid \geq \varepsilon\right\} \mid \\
& \leq \lim _{r, s \rightarrow \infty} \frac{1}{h_{r, s}}\left|\left\{(j, k) \in I_{r, s}:\left|f\left(x_{j+3, k+3}\right)-f_{N_{1}, N_{1}}\left(x_{j+3, k+3}\right)\right| \geq \frac{\varepsilon}{3}\right\}\right| \\
& +\lim _{r, s \rightarrow \infty} \frac{1}{h_{r, s}}\left|\left\{(j, k) \in I_{r, s}:\left|-3 f\left(x_{j+2, k+2}\right)+3\left(x_{j+2, k+2}\right)\right| \geq \frac{\varepsilon}{3}\right\}\right| \\
& +\lim _{r, s \rightarrow \infty} \frac{1}{h_{r, s}}\left|\left\{(j, k) \in I_{r, s}:\left|3 f\left(x_{j+1, k+1}\right)-3 f_{N_{1}, N_{1}}\left(x_{j+1, k+1}\right)\right| \geq \frac{\varepsilon}{3}\right\}\right| \\
& +\lim _{r, s \rightarrow \infty} \frac{1}{h_{r, s}}\left|\left\{(j, k) \in I_{r, s}:\left|f\left(x_{j, k}\right)-f_{N_{1}, N_{1}}\left(x_{j, k}\right)\right| \geq \frac{\varepsilon}{3}\right\}\right| \\
& +\lim _{r, s \rightarrow \infty} \frac{1}{h_{r, s}}\left|\left\{(j, k) \in I_{r, s}:\left|f_{N_{1}, N_{2}}\left(x_{j+3, k+3}\right)-3 f_{N_{1}, N_{2}}\left(x_{j+2, k+2}\right)+3 f_{N_{1}, N_{2}}\left(x_{j+1, k+1}\right)-f_{N_{1}, N_{2}}\left(x_{j, k}\right)\right| \geq \frac{\varepsilon}{3}\right\}\right| \\
& =0+0+0+0+0=0 .
\end{aligned}
$$

Corollary 2.3: The set of functions that map 2quasi-Cauchy double sequences to double lacunary statistically $\delta^{2}$ 2quasi-Cauchy double sequences in $A$ is complete in the set of continuous functions on $A$.

\section{REFERENCES}

1. H. Cakalli, On statistical convergence in topological groups. Pure Appl. Math. Sci. 43(1996), 27-31.

2. H Cakalli, Sequential definitions of Compactness, Appl. Math. Lett. 21(6), 594-598, 2008.

3. H. Cakalli and H. Kaplan, A variation on lacunary statistical quasi Cauchy sequences, commun. Fac. Sci. Univ. Ank. Series Al, 66(2), 71-79, 2017.

4. H. Cakalli and P. Das, Fuzzy compactness via summability. Journal of Applied Mathematics, 22(2009), 1665-1669.

5. L. Cheng, G. Lin, Y. Lan and H. Liu, Measure Theory of Statistical Convergence. Science in China Series A-Math. (2008) 51: 2285.

6. J. Connor, and M. A. Swardson, Strong integral summability and stone-chech compactification of the half-line. Pacific Journal of Mathematics. 157(1993), 201-224.
7. J. Connor and M. A. Swardson, Equivalence of bounded strong integral summability methods, Math. Japon. 39(1994), 401-428.

8. H. Fast, Sur la convergence statistique, Colloq. Math. 2(1951), 241-244

9. J. A. Fridy and C. Orhan, Lacunary statistical summability, J. Math, Annal. Appl. 173(1993), 497504.

10. G. Di. Maio and Lj. D. R. Kočinac, Statistical convergence in topology, Topology Appl. 156(2008), 28-45.

11. I. J. Maddox, Statistical Convergence in a locally convex space. Mathematical Proceedings of the Cambridge Philosophical Society 104(1988), 141 145.

12. H. I. Miller, A measure theoretical subsequence characterization of statistical convergence. 
Transactions of the American Mathematical Society, 347(1995), 1811-1819.

13. M. Mursaleen, and O. H. H. Edely, "Statistical convergence of double sequences," J. Math. Anal. Appl., 288(2003), 223-231.

14. F. Nuary, and E. Savas, Statistical convergence of sequences of fuzzy numbers, Math. Slovaca, 45(1995), 269-273.

15. T. Šalát, On Statistically convergent sequences of real numbers, Math. Slovaca 30 (1980), 139-150.

16. E. Savas, Statistical convergence of fuzzy numbers, Inform. Sci., 137(2001), 277-282.
17. E. Savas and R. F. Patterson, On some double almost lacunary sequence spaces defined by Orlicz functions, Filomat, 19(2005), 35-44.

18. I. J. Schoenberg, the integrability of certain functions and related summability methods. American Mathematics. Monthly 66(1959), 361375.

19. S. Yildiz, lacunary statistical delta 2 quasi Cauchy sequences, sakarya. Univ Journal of science 21(6) 1408-1412, 2017. 